\title{
Potential errors in interpreting hibernation due to FDG scaling?
}

\author{
Nils P. Johnson, MD, MS, a and K. Lance Gould, $\mathrm{MD}^{\mathrm{a}}$ \\ a Weatherhead PET Center, Division of Cardiology, Department of Medicine, McGovern Medical \\ School at UTHealth and Memorial Hermann Hospital, Houston, TX
}

Received Oct 27, 2019; accepted Oct 28, 2019

doi: $10.1007 / \mathrm{s} 12350-019-01953-\mathrm{z}$

\section{See related article, pp. 1730-1739}

Metabolic imaging of the myocardium using positron emission tomography (PET) provides a rational basis to select patients for revascularization by distinguishing between hibernating and infarcted tissues. ${ }^{1}$ As in many areas, however, the devil lies in the details. The manuscript by Degtiarova and colleagues in this issue ${ }^{2}$ provides a timely opportunity to revisit fundamental aspects of fluorodeoxyglucose F-18 (FDG) image interpretation.

After an initial 1978 investigation in humans to image myocardial glucose metabolism, ${ }^{3}$ FDG emerged in 1983 as a tool to distinguish metabolic versus perfusion patterns in patients with prior infarction. ${ }^{4}$ This foundational work from Michael Phelps and Heinrich Schelbert at the University of California in Los Angeles paved the way for a wide, subsequent literature ${ }^{5}$ leading to specific United States Food and Drug Administration (FDA) approval in 1999 of FDG for myocardial viability imaging. ${ }^{6}$ FDG provides an excellent surrogate marker of glucose uptake since it competes with glucose for intracellular transport, becomes trapped after phosphorylation by hexokinase, and does not proceed further along metabolic pathways, hence remaining fixed for myocardial imaging. ${ }^{7}$

Funding NPJ and KLG received internal funding from the Weatherhead PET Center for Preventing and Reversing Atherosclerosis.

Reprint requests: Nils P. Johnson, MD, MS, Weatherhead PET Center, Division of Cardiology, Department of Medicine, McGovern Medical School at UTHealth and Memorial Hermann Hospital, Houston, TX; Nils.Johnson@uth.tmc.edu

J Nucl Cardiol 2021;28:1740-4.

$1071-3581 / \$ 34.00$

Copyright (c) 2019 American Society of Nuclear Cardiology.
A key practical aspect of FDG imaging involves metabolic preparation. American societal guidelines recommend glucose loading after a fasting period to induce a natural insulin response and promote tracer uptake. ${ }^{7}$ In diabetic patients, the guidelines recognize the need for exogenous insulin administration and suggest either the gold standard but time-consuming euglycemic-hyperinsulinemic clamp or an abbreviated protocol using serial, intravenous, bolus insulin after a glucose load. ${ }^{8}$ Our laboratory, for example, has produced excellent results using a customized version of this latter protocol that requires achieving falling blood glucose regardless of diabetic status.

After acquisition of perfusion images (using any of the standard PET tracers and typically performed immediately before FDG imaging due to a much shorter half-life), the perfusion and metabolic patterns must be compared against each other to classify the result into four options: matched normal (normal myocardium with intact perfusion and glucose metabolism), matched abnormal (infarcted myocardium with perfusion defect and reduced/absent glucose metabolism), mismatch (hibernating myocardium with perfusion defect but intact glucose metabolism), and reverse mismatch (viable myocardium with normal perfusion but reduced/ absent glucose metabolism). While commonly interpreted visually, how exactly should these two images be compared quantitatively? Addressing this basic question reveals potential errors of both interpretation and quantification inconsistent with known myocardial physiology.

\section{BEST PERFUSION = NORMAL GLUCOSE METABOLISM?}

For quantitative analysis of FDG images, several groups assume that the most normally perfused area corresponds to the region with highest glucose metabolism. In the current work by Degtiarova and colleagues, 
${ }^{، 18}$ F-FDG uptake was expressed as percentage of the segment with the highest mean late ${ }^{13} \mathrm{~N}-\mathrm{NH}_{3}$ uptake, assuming that the segment with the highest 'perfusion' should have normal metabolism.",2 This scaling mirrors the landmark PARR-1 study, "To normalize the FDG to perfusion, sectors that were $\geq 80 \%$ of the maximum perfusion were defined as normal. FDG uptake was then normalized by scaling the percent FDG uptake to be equal to perfusion in the 'maximum zone' sectors.", How valid is this assumption?

At first glance, neither of these proposals ${ }^{2,9}$ matches the language of the American societal guidelines that "the comparison of perfusion and metabolism images obtained with PET is relatively straightforward because both image sets are attenuation corrected, ${ }^{, 7}$ implying no need to modify the respective relative uptake images. But research often leads guidelines, so perhaps the "straightforward" might be straight but not forward thinking.

Using preliminary data from viability patients imaged at our center over the past 8 years, we explored this assumption by comparing the relative uptake of the most normally perfused region ( $\geq 80 \%$ of maximum) to the relative uptake of the corresponding pixels on the FDG relative images. Patients with a ratio of exactly 1.0 imply a perfect match whereby best perfusion indeed reflects best glucose metabolism. However, patients with a ratio not equal to 1.0 indicate a disconnect between the areas of best perfusion and best glucose metabolism. A patient with relative perfusion-to-FDG ratio of $>1.0$ signals an FDG "hot spot", outside of the relative highest, most normally perfusion region; a patient with a relative perfusion-to-FDG ratio of $<1.0$ corresponds to FDG uptake that exceeds the average, normal relative perfusion.

As visual examples, Figure 1 contrasts representative short-axis images of rest and FDG relative uptake for cases with ratios of 1.8 (best FDG uptake outside of the most normally perfused region) and 0.97 (best FDG uptake exceeds average, normal perfusion). In addition to the raw, relative images, we provide rescaled FDG images of the same PET study matched to the most normally perfused region. Scaling the FDG image in this manner changes the interpretation towards more hibernating myocardium (in the case of a ratio $>1$ ) or less hibernating myocardium (in the case of a ratio $<1$ ).

To add quantitative heft to these examples, in our cohort of 318 viability scans performed primarily with rubidium- 82 as the perfusion tracer, the median primary raw rest perfusion/FDG ratio reached 1.26 (interquartile range 1.13 to 1.45 ). Just under $2 \%$ of cases (6 of 318 ) had a ratio less than 1.0, indicating that the vast majority of patients exhibit their best FDG uptake in a different region than normal perfusion. While $79 \%$ of cases $(251$ of 318) had a ratio between 1.0 and 1.5, implying similarly matched normal areas for perfusion and FDG, $19 \%$ had a ratio of 1.5 or greater with a maximum of almost 3.8. Therefore in approximately 1 of 5 patients, the areas of best perfusion and highest glucose metabolism do not correspond.

Do these severe violations of the " best perfusion = highest glucose metabolism" assumption arise due to recalcitrant diabetics? Overall, we found no significant difference in the prevalence of diabetes above versus below a rest perfusion/FDG 1.5 threshold (39\% vs $48 \%$, $\chi^{2} P$ value .302). Furthermore, the distribution of rest perfusion/FDG ratios did not differ significantly by the presence or absence of diabetes (median 1.29 vs 1.24 , Wilcox $P$ value .118), outpatient use of insulin among those with diabetes (median 1.29 vs $1.29, P$ value .645 ), or insulin-dependent diabetics versus all others (median 1.29 vs $1.25, P$ value .199).

Thus, the disconnect between baseline perfusion and glucose metabolism cannot be explained by diabetes or metabolic preparation. Instead, it reflects fundamental physiology. Nothing about myocardial metabolism demands that the most normally perfused region will have the best glucose uptake. In fact, it would be reasonable to expect that it is "harder" to switch normally perfused myocardium from fatty acids to glucose since fatty acids are the preferred energy source of the myocardium except under extreme conditions of ischemia or insulin stimulation. Indeed, our observation that almost all patients have a rest perfusion/FDG ratio $>1$ supports this incomplete conversion with intact perfusion.

Reduced glucose uptake in a normally perfused region, like the left-hand example in Figure 1, simply implies the existence of other metabolic substratesmainly fatty acids, but also lactate, amino acids, and ketone bodies. ${ }^{10}$ From a physiologic perspective, scaling glucose uptake to match the area of best perfusion produces a biased estimate of substrate utilization by artificially enhancing apparent glucose utilization. From an imaging perspective, scaling glucose uptake to match the area of best perfusion implies accepting a lower amount of glucose uptake in order to classify a segment as hibernating, thereby reducing the accuracy to predict improved LV function after revascularization.

\section{NON-ISCHEMIC CARDIOMYOPATHY}

The preceding discussion and indeed the development of FDG as a viability tracer have focused on patients with significant coronary disease and prior infarction. But what about its application to patients without either feature? The manuscript by Degtiarova and colleagues in this issue ${ }^{2}$ explores the patterns of 


\section{Best glucose outside of best perfusion}

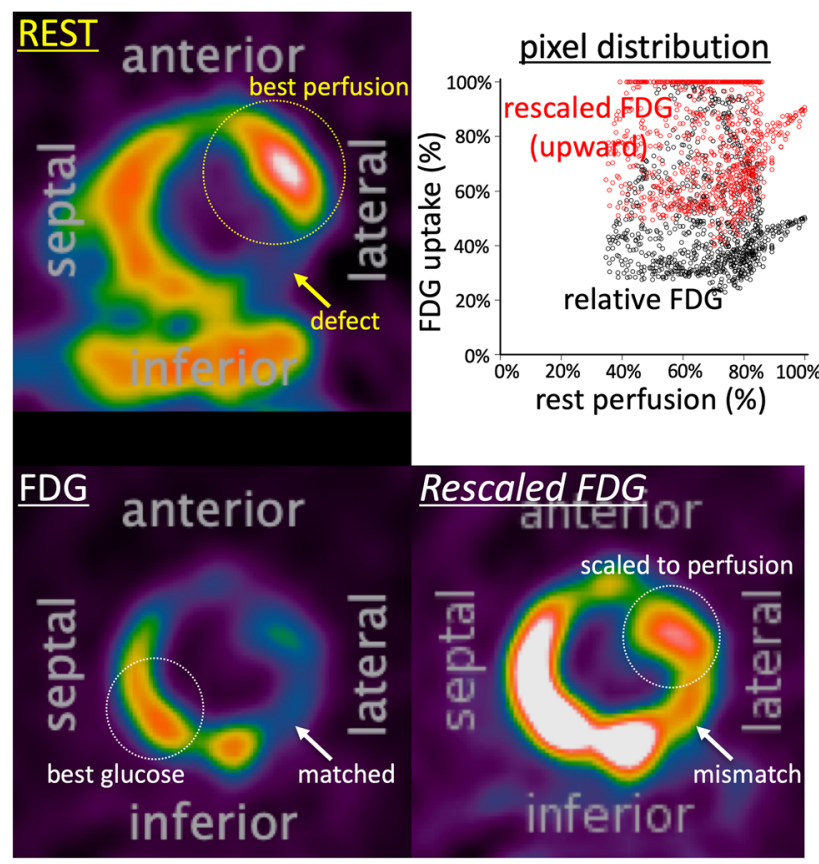

\section{Above average glucose with best perfusion}

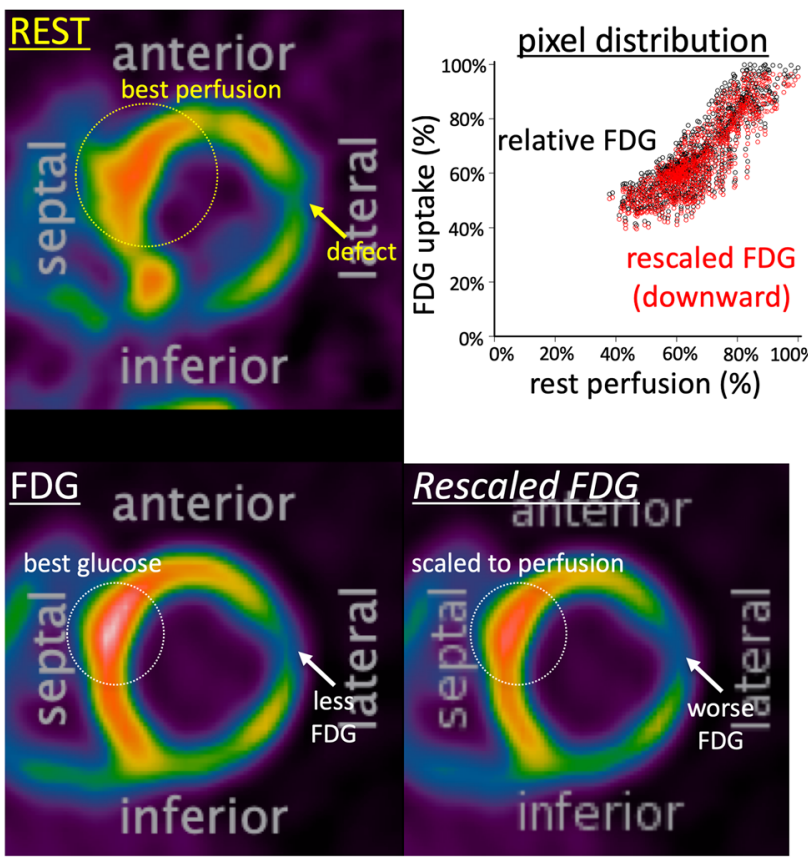

Figure 1. Examples of rescaling on image interpretation. Two examples showing representative relative uptake images of the left ventricle in short axis at rest (using rubidium-82 as perfusion tracer) and corresponding glucose uptake (using fluorodeoxyglucose [FDG] F-18 as metabolic tracer). Each example highlights the areas of best perfusion and best metabolism. The scatterplot shows the relative perfusion uptake and relative glucose metabolism using raw pixel data (black circles) and rescaled glucose data (red circles). Rescaled glucose images match the average uptake in the most normally perfused pixels ( $80 \%$ or greater relative perfusion). (Left) Because the areas of best perfusion (anterolateral) and best glucose uptake (inferoseptal) do not align (perfusion/FDG ratio of 1.8 in the most normally perfused area), rescaling moves the FDG pixels upward. Visually the matched inferolateral perfusion defect now appears mismatched, incorrectly suggesting hibernation when using the rescaled FDG images. (Right) Because the area of best perfusion has a lower average uptake than the area of best glucose metabolism (both anteroseptal), rescaling moves the FDG pixels downward. The moderate lateral perfusion defect with less FDG uptake becomes even worse after rescaling, incorrectly suggesting even worse reverse mismatch.

resting perfusion (using $\mathrm{N}-13$ ammonia) and glucose uptake (using FDG) in a cohort of 25 subjects with nonischemic heart failure and left bundle branch block (LBBB) about to undergo cardiac resynchronization therapy (CRT).

The authors' method of analyzing N-13 ammonia as a perfusion tracer complicates the analysis due to its time-dependent intracellular trapping via the glutamine pathway and subsequent exit from myocardial cells. ${ }^{11}$ While quantitative perfusion models can account for its movement into and out of this "metabolic compartment," the late relative uptake images mix perfusion and metabolism. Their 20-minute acquisition for relative uptake starting 20 minutes after $\mathrm{N}-13$ ammonia injection exceeds the recommended 10 to 15 minutes of imaging starting 1.5 to 3 minutes after tracer injection. ${ }^{7}$ Using a very late perfusion image to rescale the FDG image further exacerbates the primary rescaling problem above, as admitted by the authors since "late ${ }^{13} \mathrm{~N}-\mathrm{NH} 3$ uptake represents a combination of perfusion and metabolism (probably amino acid related) rather than pure myocardial perfusion." ${ }^{, 2}$ Thus, the imaging protocol mixes the extravascular and metabolic compartments, loses the relative lateral wall/septal perfusion differences that exacerbate the rescaling problem, and thereby distorts the regional perfusion/FDG ratio as to invalidate any interpretation of viability or perfusion-metabolism-workload relations. Their own data therefore indicate flawed methodology of using late N-13 ammonia images for 


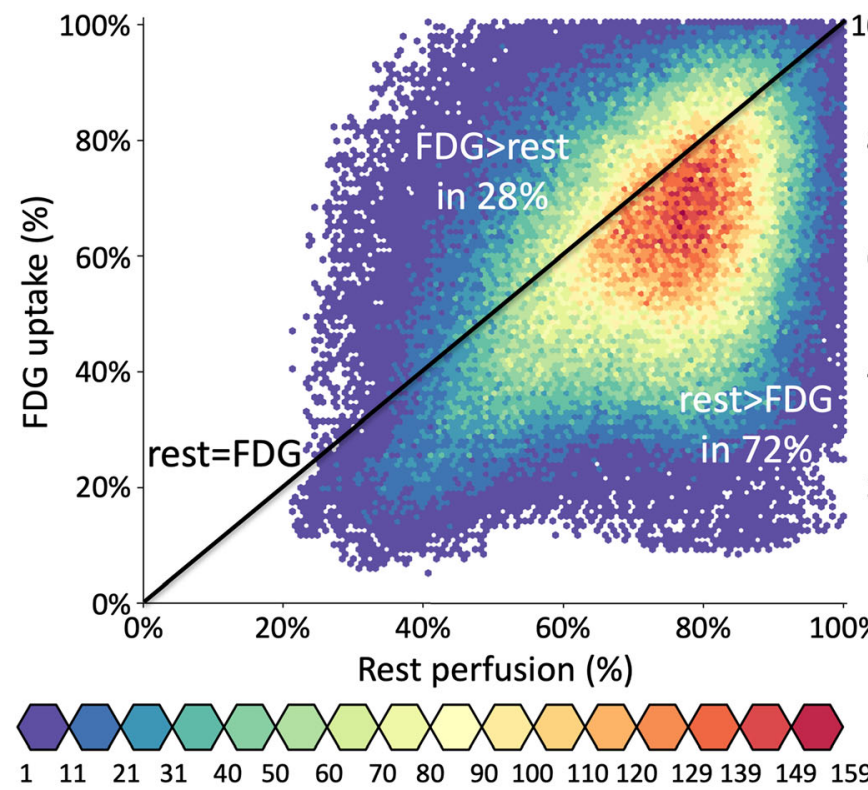

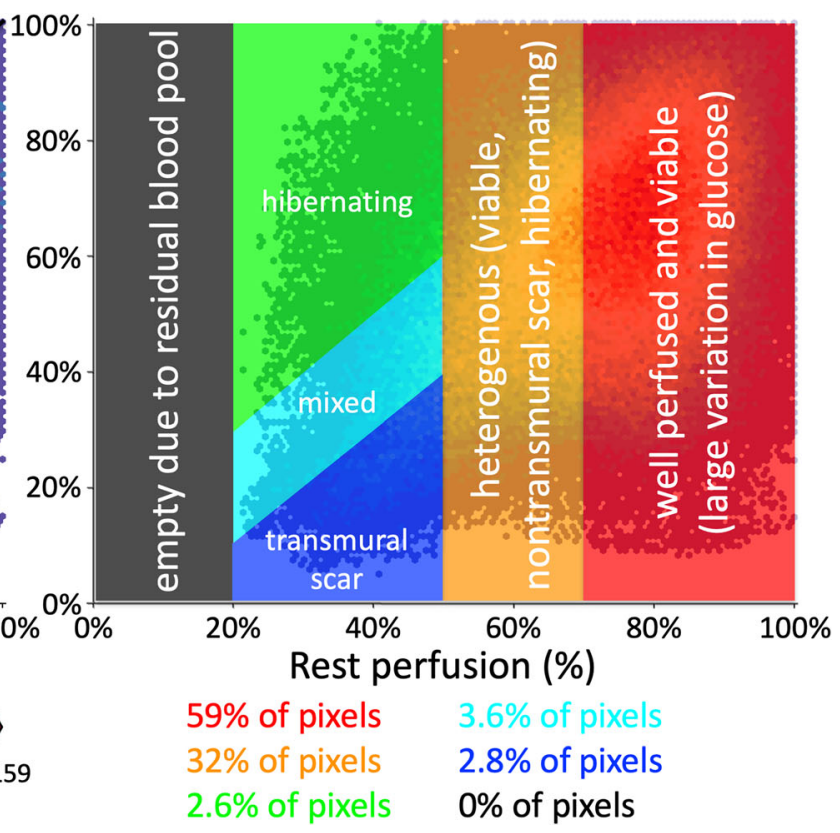

Figure 2. Distribution of rest perfusion and glucose metabolism. A two-dimensional "heat map" from approximately 300,000 pixels in 318 patients. As can be seen from the line of identity, most regions of the heart display better perfusion than glucose metabolism. By delineating regions, although the distribution is clearly continuous and therefore such borders are to some extent arbitrary, it becomes apparent that almost $60 \%$ of pixels are well perfused and viable. Even in an enriched cohort selected for potential viability, $<10 \%$ of pixels display a severe perfusion defect with classic glucose mismatch.

comparison to FDG images rather than any new insight into physiology.

Finally, their application of FDG imaging to a cohort without significant coronary disease or a history of ischemic heart disease raises other mechanisms for the observations since the terms "match" and "mismatch" derive from classic ischemic/infarct cardiomyopathy. What does relative glucose uptake teach us with sufficient epicardial delivery of nutrients but intrinsic myocyte dysfunction? This issue adds to the profound error of rescaling FDG images and using nonperfusion late N-13 images for comparison to FDG images, thereby warranting skepticism regarding their claims of perfusion/metabolism patterns.

\section{PERSONALIZED VIABILITY INTERPRETATION}

In order to assess how scaling affects interpretation of combined resting perfusion and glucose uptake images, we compiled pixel-level data from our 318 patient cohort for a total of approximately 300,000 points (21 slices and 64 radial pixels per scan but excluding the basal 4 and apical 2 slices and also masking the membranous septum). Figure 2 presents the two-dimensional distribution of these pairings and allows for several conclusions. First, a $72 \%$ majority of pixels have lower FDG uptake than resting perfusion, further emphasizing that most regions of the heart do not completely convert to glucose metabolism despite metabolic preparation, especially when flow remains intact. Second, while a direct correlation exists between perfusion and FDG, it is weak with a Pearson $r=0.38$ $(P<.0001)$, indicating that only $0.38^{2}=14 \%$ of the variation in FDG uptake can be explained by resting perfusion and the other $86 \%$ represents biologic heterogeneity of metabolic substrate utilization, referral patterns for viability imaging, and a myriad of other modifying factors. Third, resting perfusion does not fall below $20 \%$ relative to maximum due to residual blood pool activity, whereas FDG enters this range due to good-quality metabolic preparation. Fourth, any boundaries on Figure 2 must represent artificial categories since the distribution is continuous.

Visual analysis of these figures suggests broad groupings that correspond to the major categories of perfusion-FDG comparisons as follows: well perfused, viable myocardium (relative perfusion $70 \%$ to $100 \%$ of maximum); heterogenous myocardium composed of variable admixtures of viable tissue, non-transmural scar, and hibernating myocardium (relative perfusion $50 \%$ to $70 \%$ of maximum); severely reduced perfusion with FDG uptake identifying hibernating myocardium 
from transmural scar (relative perfusion $20 \%$ to $50 \%$ of maximum) of primary clinical importance; and an empty region due to residual blood pool activity (relative perfusion $0 \%$ to $20 \%$ of maximum).

In summary, the data of the current manuscript and our large patient cohort analyzed for pixel distribution of relative perfusion and FDG uptake indicate that rescaling FDG images to the most normally perfused area is not correct, not physiologic, and will bias interpretation of FDG imaging toward more viability than actually present, hence potentially leading to non-beneficial, high risk-revascularization procedures. These observations warrant further quantification to understand their clinical outcomes as modified by revascularization in existing data like PARR-1, ${ }^{9}$ the ongoing AIMI-HF trial (clinicaltrials.gov NCT01288560), and future protocols.

\section{Disclosures}

NPJ and KLG have pending patents on diagnostic methods for quantifying aortic stenosis and TAVI physiology, and on algorithms to correct pressure tracings from fluid-filled catheters. NPJ has an institutional licensing and consulting agreement with Boston Scientific for the smart minimum FFR algorithm, and has received significant institutional research support from Philips Volcano Corporation (DEFINE-FLOW, NCT02328820) and St. Jude Medical (CONTRAST, NCT02184117) for studies using intracoronary pressure and flow sensors. $K L G$ is the $510(k)$ applicant for CFR Quant (K113754) and HeartSee (K143664 and K171303), software packages for cardiac positron emission tomography image processing, analysis, and absolute flow quantification.

\section{References}

1. D'Egidio G, Nichol G, Williams KA, Guo A, Garrard L, deKemp $\mathrm{R}$, et al. Increasing benefit from revascularization is associated with increasing amounts of myocardial hibernation: a substudy of the PARR-2 trial. JACC Cardiovasc Imaging 2009;2:1060-8.

2. Degtiarova G, Claus P, Duchenne J, Schramm G, Nuyts J, Verberne HJ, et al. Impact of left bundle branch block on myocardial perfusion and metabolism: A positron emission tomography study. J Nucl Cardiol 2019. https://doi.org/10.1007/s12350-019-01900-y.

3. Phelps ME, Hoffman EJ, Selin C, Huang SC, Robinson G, MacDonald N, et al. Investigation of [18F]2-fluoro-2-deoxyglucose for the measure of myocardial glucose metabolism. J Nucl Med 1978;19:1311-9.

4. Marshall RC, Tillisch JH, Phelps ME, Huang SC, Carson R, Henze $\mathrm{E}$, et al. Identification and differentiation of resting myocardial ischemia and infarction in man with positron computed tomography, 18F-labeled fluorodeoxyglucose and $\mathrm{N}-13$ ammonia. Circulation 1983;67:766-78.

5. Tillisch J, Brunken R, Marshall R, Schwaiger M, Mandelkern M, Phelps M, et al. Reversibility of cardiac wall-motion abnormalities predicted by positron tomography. N Engl J Med 1986;314:8848.

6. Raczkowski VFC. Medical review of F-18 fluorodeoxyglucose positron emission tomography (F-18 FDG PET) for cardiac indications. https://www.fda.gov/media/77638/download. Accessed October 21, 2019.

7. Dilsizian V, Bacharach SL, Beanlands RS, Bergmann SR, Delbeke $\mathrm{D}$, Dorbala $\mathrm{S}$, et al. ASNC imaging guidelines/SNMMI procedure standard for positron emission tomography (PET) nuclear cardiology procedures. J Nucl Cardiol 2016;23:1187-226.

8. Martin WH, Jones RC, Delbeke D, Sandler MP. A simplified intravenous glucose loading protocol for fluorine-18 fluorodeoxyglucose cardiac single-photon emission tomography. Eur J Nucl Med 1997;24:1291-7.

9. Beanlands RS, Ruddy TD, deKemp RA, Iwanochko RM, Coates G, Freeman M, et al. Positron emission tomography and recovery following revascularization (PARR-1): The importance of scar and the development of a prediction rule for the degree of recovery of left ventricular function. J Am Coll Cardiol 2002;40:1735-43.

10. Ritterhoff J, Tian R. Metabolism in cardiomyopathy: every substrate matters. Cardiovasc Res 2017;113:411-21.

11. Bergmann SR, Hack S, Tewson T, Welch MJ, Sobel BE. The dependence of accumulation of $13 \mathrm{NH} 3$ by myocardium on metabolic factors and its implications for quantitative assessment of perfusion. Circulation 1980;61:34-43. 